\title{
Structure and magnetic properties of Co chains on a stepped Cu surface
}

\author{
$\check{S}_{\text {Pick }}^{1}$, P A Ignatiev ${ }^{2,4}$, A L Klavsyuk ${ }^{2}$, W Hergert ${ }^{3}$, V S Stepanyuk ${ }^{2}$ and \\ P Bruno ${ }^{2}$ \\ ${ }^{1}$ J Heyrovský Institute of Physical Chemistry of the Academy of Sciences of the Czech Republic, \\ v.v.i., Dolejškova 3, CZ-182 23 Prague 8, Czech Republic \\ 2 Max-Planck-Institut für Mikrostrukturphysik, Weinberg 2, D-06120 Halle, Germany \\ ${ }^{3}$ Institut für Physik, Martin-Luther-Universität, Halle-Wittenberg, Friedemann-Bach-Platz 6, \\ D-06099 Halle, Germany \\ E-mail: ignatiev@mpi-halle.de
}

Received 19 June 2007, in final form 17 July 2007

Published 24 September 2007

Online at stacks.iop.org/JPhysCM/19/446001

\begin{abstract}
Magnetic moments and the magnetic anisotropy energy (MAE) are calculated for $\mathrm{Co}$ chains on a stepped $\mathrm{Cu}(111)$ surface in a fully relaxed geometry. The Korringa-Kohn-Rostoker Green's function method is used to determine parameters of $N$-body interatomic potentials and to fit the semi-empirical tightbinding electronic Hamiltonian. The strain relief at steps and in the Co chains is demonstrated to have a profound effect on the morphology of the substrate. Atomic relaxations are shown to decrease the magnetic moments and the MAE. The MAE and orbital moments are found to exhibit an oscillatory behavior with increasing size of the chains.
\end{abstract}

(Some figures in this article are in colour only in the electronic version)

\section{Introduction}

Since the pioneering work of Elmers et al [1] on Fe nanostripes on a W(110) surface, a large number of experimental and theoretical studies have been devoted to quasi-one-dimensional (1D) systems on different substrates [2-16]. A question of primary interest is related to the magnetic properties of low-dimensional systems. Such nanostructures are considered as possible candidates for future magnetoelectronic device use: magnetic properties of lowdimensional systems can be very much different from properties of bulk materials. For example, the orbital contribution to magnetism can be strongly enhanced at surfaces as was shown by Ticher et al for $\mathrm{Co}$ on $\mathrm{Cu}(001)$ [14]. According to their experimental and theoretical studies, the orbital moment of the first Co layer was twice larger than in the bulk. The enhanced

4 Author to whom any correspondence should be addressed. 
spin magnetic moment in the surface layer and reduced spin magnetic moment in a subsurface layer of $2 \mathrm{Co} / \mathrm{Cu}(001)$ have been obtained in ab initio calculations of Pentcheva et al [17]. Strong relaxation of the interlayer spacing has been shown to affect magnetic moments. FelixMedina et al [15] performed tight-binding (TB) calculations which revealed remarkably large total magnetic moment of Co wires on $\operatorname{Pd}(110)$. They have shown that the orbital moment per Co atom in wires is almost three times larger than the Co bulk orbital moment. Dürr et al [18] reported on similar results for Co films on a stepped $\mathrm{Cu}\left(\begin{array}{lll}1 & 1 & 13\end{array}\right)$ surface. TB calculations of Dorantes-Dávila and Pastor [5] have predicted that the MAE of 3d transition metal wires can be an order of magnitude larger than in two-dimensional thin films [5]. Experiments revealed extremely large MAE values for Co adatoms, nanoparticles and Co wires on $\operatorname{Pt}(111)[4,19,20]$. On the other hand, the full-potential linearized augmented plane wave (FLAPW) calculations performed by Hong and $\mathrm{Wu}[8,9]$ revealed no obvious MAE enhancement in Co chains in comparison to Co thin films. The MAE and orbital magnetic moments were found to exhibit an oscillatory behavior with increasing size of the chains. Magnetic moments of supported monatomic $\mathrm{Co}$ chains on $\mathrm{Cu}(001)$ and $\mathrm{Pt}(001)$ were found to align along the chain, contrary to the perpendicular alignment of the magnetic moment of the same free-standing chains. Calculations for spin and orbital moments of finite Co nanowires on $\mathrm{Cu}(001)$ performed by Klautau and Frota-Pessôa [21] by means of the linear muffin tin orbital atomic approximation (LMTO) method have predicted orbital moments of $0.7 \mu_{\mathrm{B}}$ for the Co sites at the end of a Co nanowire on $\mathrm{Cu}(001)$, and $0.3 \mu_{\mathrm{B}}$ per atom in the infinite $\mathrm{Co}$ wire on $\mathrm{Cu}(001)$. These values should be experimentally observable. Lazarovits et al [12, 13, 22] have studied magnetic properties of finite monatomic Fe chains on $\mathrm{Cu}(001)$ and $\mathrm{Cu}(111)$ by means of Korringa-KohnRostoker (KKR) Green's function method. They have found that spin and orbital moments in the chains are larger than those in the monolayer. A strong out-of-plane easy axis of magnetization was found for chains on $\mathrm{Cu}(001)$ and $\mathrm{Cu}(111)$.

Stepped surfaces bring an additional magnetic anisotropy within the surface plane since the direction parallel and perpendicular to the step edge are no longer equivalent [23]. Dhesi et al [24] have found by means of x-ray magnetic linear dichroism measurements that the spinorbit coupling parallel to the step is larger than that perpendicular to the step. Weber et al [25] have reported on the adatom-induced reorientation of spins in $\mathrm{Co}$ on a stepped $\mathrm{Cu}$ surface from the direction parallel to steps to the perpendicular one. Hahlin et al [26] have performed x-ray magnetic circular dichroism (XMCD) and x-ray reflectivity measurements on Co deposited on vicinal and flat $\mathrm{Cu}(111)$. They have revealed that the orbital moment and the spin moment for the vicinal surface are enhanced by $25 \%$ for the thickness range 1-25 monolayers as compared to those found for Co on the flat surface. The first-principles investigation of Shick et al [27] of the anomalous ferromagnetism of a quasi-one-dimensional Co chains at the $\operatorname{Pt}(111)$ step edge has shown that the symmetry breaking at the step leads to an easy magnetization axis at an odd angle of about $20^{\circ}$ towards the Pt step.

Still little attention has been paid so far to the effect of strain relaxations at steps on structure and magnetic properties of chains. There have been only a few investigations, to our knowledge, which have addressed this problem for infinite chains. A $b$ initio calculations of Máca et al [28] have indicated that the effect of structural relaxations on magnetic properties of infinite Co wires on a stepped Pt surface could be important to correctly describe the MAE on stepped surfaces [28]. Spišák and Hafner [10, 11] have found by use of a PAW package that the equilibrium bond length of a free-standing straight wire is smaller by $12 \%$ than the corresponding bond length of a wire on a $\mathrm{Cu}$ substrate. These results indicate that wires on a surface are under a strong tensile stress. The aim of our work is to study the strain relaxations in finite atomic chains at stepped surfaces and their effect on magnetic properties. Performing $a b$ initio and TB calculations, we concentrate on $\mathrm{Co}$ chains on a stepped $\mathrm{Cu}(111)$ surface. 


\section{Computational methods}

$A b$ initio calculations for ideal $\mathrm{Co}$ chains on a stepped $\mathrm{Cu}(111)$ surface are performed by KKR and Screened KKR Green's function method (SKKR) [29, 30] in the framework of the density functional theory (DFT) in the local spin density approximation (LSDA). Up to several hundreds of inequivalent atoms can be involved in self-consistent calculations. The concept of a vicinal surface is applied to simulate a superlattice of regularly separated steps of monatomic height $[31,32]$. A stepped surface is treated as a two-dimensional perturbation of the infinite bulk. The Dyson equation for stepped surfaces is solved in $k_{\|}$representation, where $k_{\|}$belongs to 2D Brillouin zone. Finite chains adsorbed at the step edges destroy the $2 \mathrm{D}$ periodicity. Therefore, we calculate electronic and magnetic properties of chains in a real-space representation. In this case, the Green's function of a stepped surface (we have chosen $\mathrm{Cu}(554)$ surface) calculated by means of the SKKR method is used in the Dyson equation as the reference Green's function. Details of our approach can be found elsewhere [29-31].

The relaxed atomic geometries have been obtained by means of ab initio fitted manybody potentials formulated in the second moment approximation of the TB theory [33-35]. The KKR Green's function method $[29,30]$ is used to create an ab initio data pool to fit the parameters of the potentials. Surface (binding energies of supported Co clusters of different sizes, the Hellmann-Feynman forces acting on the Co adatom for different positions above the surface) and bulk properties (bulk modulus, lattice constants, cohesive energies, and elastic constants) are included in the data pool. The reliability of such potentials for different atomic configurations has been demonstrated [36-42]. Recent studies [43] have shown that our method describes atomic relaxations in nanostructures in very good agreement with fully $a b$ initio calculations. Note, a full optimization of the structure of chains, steps and the substrate by $a b$ initio methods is still beyond the accessible computation time. For technical details we refer to our former studies [35].

In order to avoid tedious first-principles electronic structure calculations for relaxed atomic geometries and to include spin-orbit coupling we apply the TB method for relaxed chains. To make the extensive calculations feasible, only the changes introduced by the geometry relaxation of the Co atoms and their nearest neighbors are taken into account. It has been demonstrated in our previous studies $[38,44]$ that moderate perturbation of more distant $\mathrm{Cu}$ atom can be safely neglected. The parameters of a semi-empirical TB Hamiltonian are fitted to reproduce closely the $a b$ initio KKR results for ideal chains at the step (atomically resolved local density of states (LDOS) and magnetic moments). TB calculations are performed selfconsistently with commonly used conditions: the local charge neutrality and linear dependence of the exchange splitting on the spin magnetic moment [45]. The local densities of states (LDOS) and electronic properties of interest are calculated with the help of the recursion method technique (continued fraction with large number of levels) [46, 47]. Actually, the method is essentially the same as in our previous investigations [38, 44]. In order to avoid too sharp peaks near $E_{\mathrm{F}}$ we have changed the small $d d \delta$ parameter of Co [48] to fulfill the canonical prescription [49] $d d \sigma: d d \delta=6: 1$. This minor change in the parameterization seems to have no effect on the results.

\section{Results and discussion}

Relaxation of short $\mathrm{Co}$ chains placed at the $\mathrm{Cu}(111)$ vicinal surface step edge would differ significantly from the case of the flat $\mathrm{Cu}(111)$ substrate due to interactions of $\mathrm{Co}$ atoms with the $\mathrm{Cu}$ atoms of the step. We calculated relaxations of short $\mathrm{Co}_{2}-\mathrm{Co}_{7}$ chains at the step edge 


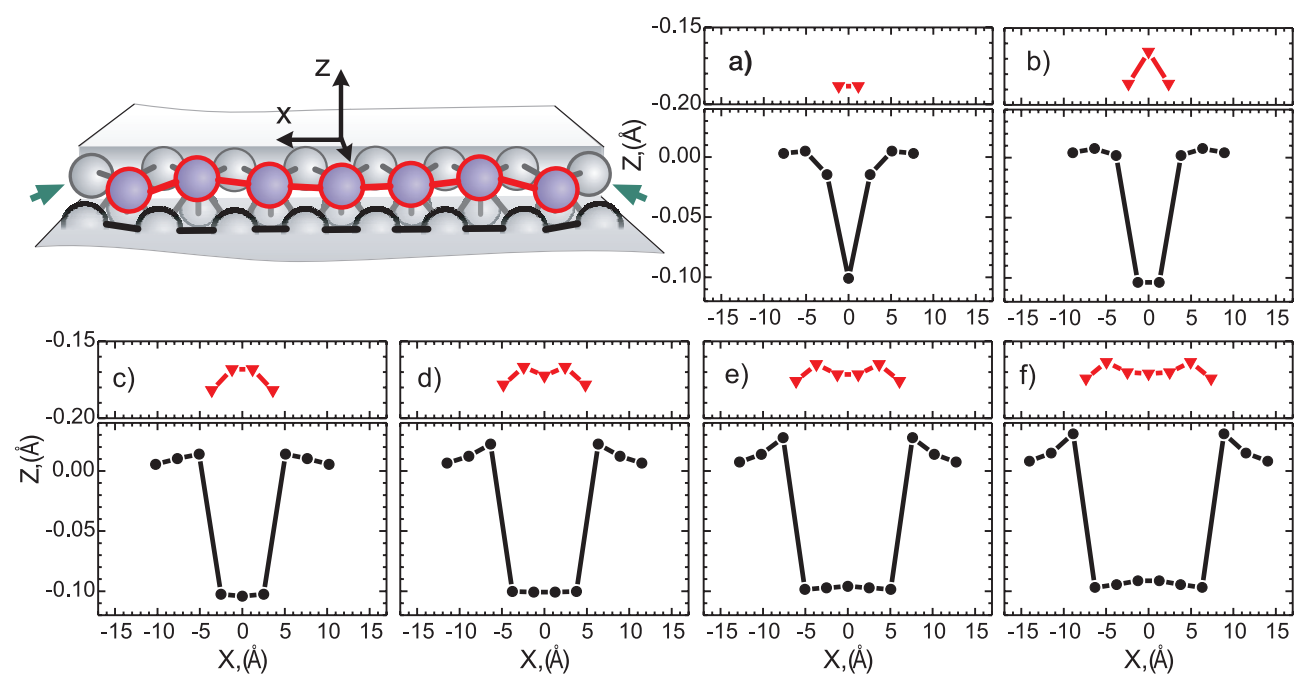

Figure 1. Strain relaxations in short $\mathrm{Co}$ chains placed at the $\mathrm{Cu}(111)$ vicinal surface step edge. The upper panels of all the figures demonstrate displacements of $\mathrm{Co}$ atoms in (a) $\mathrm{Co}_{2}$, (b) $\mathrm{Co}_{3}$, (c) $\mathrm{Co}_{4}$, (d) $\mathrm{Co}_{5}$, (e) $\mathrm{Co}_{6}$, (f) $\mathrm{Co}_{7}$ chains. The corresponding relaxation profiles of underlying substrate atoms are shown in lower panes. Coordinate origins coincide with the geometrical centers of corresponding unrelaxed structures.

of $\mathrm{Cu}(554)$ vicinal surface. The coordinate frame used is drawn in the inset of figure 1 . The $x$ axis is parallel to the step, the $z$-axis direction matches the surface terrace normal and the $y$ axis points from the step. Projections of relaxed atomic positions of the Co chains and underlying substrate atoms on $x z$ plane are plotted respectively in upper and lower panels of figures 1(a)(f). Vertical displacements of Co atoms strongly depend on the chain length. To rationalize such a difference and to find common features in Co chain relaxations, we should stress that the edge atoms of Co chains due to lower coordination number, exhibit the strongest relaxations towards the substrate, towards the step and towards the adjacent $\mathrm{Co}$ atom. The positions of $\mathrm{Cu}$ atoms in the step remain practically the same except when at the chain edges (they are marked with green arrows in the inset of figure 1). These atoms move out from the step to the terrace. The picture described above remains valid for all the considered Co chains (see upper panels of figures 1(a)(f)). Such a displacement of the edge Co atoms hinders the relaxation of the second atom in the chain towards the substrate. As a result, the second Co atoms are situated remarkably higher than the edge ones. This 'edge effect' completely explains the arched relaxations profiles of $\mathrm{Co}_{3}$ and $\mathrm{Co}_{4}$ chains. The longer $\mathrm{Co}_{5}, \mathrm{Co}_{6}$ and $\mathrm{Co}_{7}$ chains have the 'letter M-like' shape when three outermost Co atoms form two arches at each edge of the chain and the remaining Co atoms in the center exhibit similar relaxations forming steady central region. The description of the atomic relaxations in the Co chains can be summarized in the $3 \mathrm{D}$ illustration as it is plotted in figure 2 for the $\mathrm{Co}_{7}$ chain. All the considered Co chains push underlying substrate atoms downwards, but the outer substrate atoms exhibit upward vertical relaxations due to the shift of the edge Co atoms towards the chain center. The relative changes of $\mathrm{Co}-\mathrm{Co}$ and $\mathrm{Co}-$ $\mathrm{Cu}$ bonds in $\mathrm{Co}_{7}$ chain caused by relaxation are listed in table 1. The Co atoms' enumeration is explained in figure 3. Because of the reflection symmetry $x \rightarrow-x$ only the first four Co atoms are considered in table 1 . The zoomed view in figure 3 clarifies the bond notation. To illustrate the effect of relaxations on the electronic structure of the $\mathrm{Co}_{7}$ chain we also put in table 1 values of spin magnetic moments for ideal and relaxed configurations. In both cases 


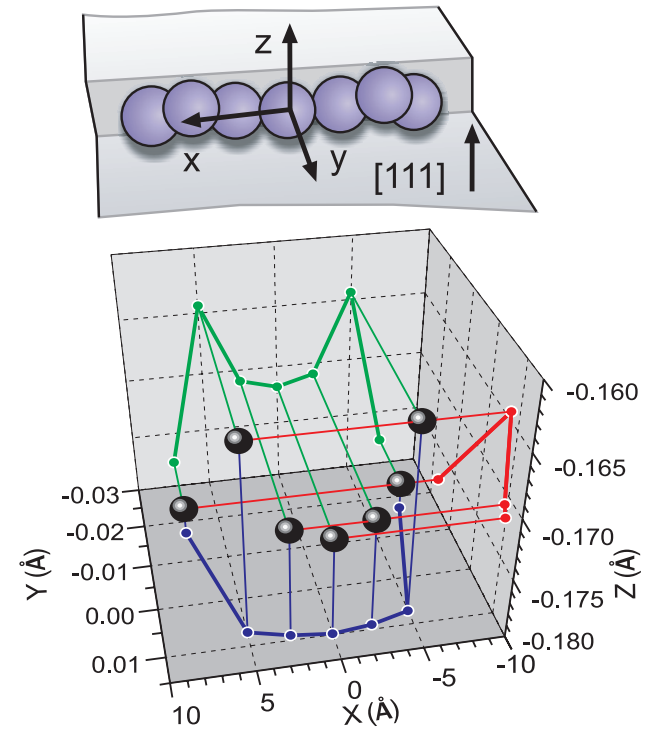

Figure 2. The three-dimensional relaxation profile in the $\mathrm{Co}_{7}$ chain. Edge atoms are strongly attracted to the step due to the lower coordination number. The second atoms from the edge are shifted from the step. Atoms in the middle of the chain lie approximately in a straight line.

Table 1. Effect of atomic relaxations on structural and magnetic properties of the $\mathrm{Co}_{7}$ chain. Values of $\mathrm{Co}-\mathrm{Co}$ and $\mathrm{Co}-\mathrm{Cu}$ bonds length changes are listed in per cents relative to the unrelaxed bond length. Order of the Co atoms and bonds notation are illustrated in figure 3.

\begin{tabular}{lrrrc}
\hline \multirow{5}{*}{ Bonds } & \multicolumn{5}{c}{ Co atom } \\
\cline { 2 - 5 } & 1 & 2 & 3 & 4 \\
\hline 1 & - & -6.2 & -3.2 & -2.3 \\
2 & -6.2 & -3.2 & -2.3 & -2.3 \\
3 & 0.3 & 0.6 & -0.8 & -1.5 \\
4 & -5.2 & -2.6 & -2.0 & -1.5 \\
5 & -0.8 & -0.4 & -1.9 & -2.6 \\
6 & -6.1 & -3.8 & -3.2 & -2.6 \\
7 & -3.5 & -3.3 & -3.3 & -3.3 \\
\hline \multicolumn{5}{c}{ Magnetic moments $\left(\mu_{\mathrm{B}}\right)$} \\
Ideal & 1.71 & 1.68 & 1.64 & 1.66 \\
Relaxed & 1.59 & 1.52 & 1.53 & 1.50 \\
\hline
\end{tabular}

edge Co atoms have the maximum magnetic moment in the chain. Atomic relaxations reduce magnetic moments of all the atoms.

Some general discussion on the symmetries in the considered systems is required. The presence of the reflection symmetry $x \rightarrow-x$ fixes the $x$-direction (parallel to the chain) for the magnetic anisotropy and therefore the corresponding orbital and spin moments are parallel. But it is still necessary to perform a complete analysis of the magnetic anisotropy in $y z$ plane. Strictly speaking, the magnetic $\operatorname{spin}^{5}$ and the orbital moments need not to be

5 We identify the spin moment by the direction $\mathbf{n}$ included in the part of the TB Hamiltonian $0.5 \Delta(\mathbf{n} \boldsymbol{\sigma})$ introducing the exchange splitting $\Delta$. 


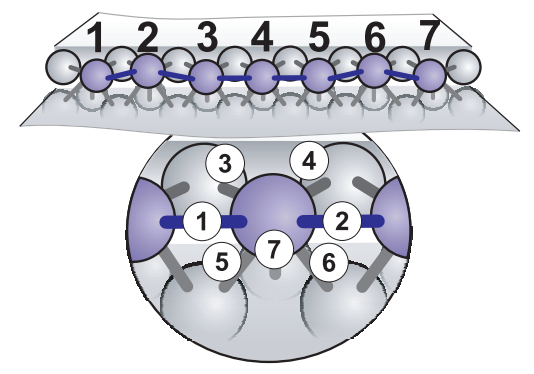

Figure 3. Sketch of the $\mathrm{Cu}(111)$ vicinal surface step with the $\mathrm{Co}_{7}$ chain. Substrate $\mathrm{Cu}$ atoms are drawn with grey colour; the Co chain is light blue. The top view illustrates the Co atoms' enumeration order used in table 1 . The bottom zoomed view is designed to clarify the bond notation used in table 1 .

mutually parallel [50], so we vary the orientation of the spin moment in the $y z$ plane and for each spin moment orientation we calculate the orbital moment projections onto $y$ and $z$ axes. The obtained orbital moments and anisotropy energies are fitted by the formula

$$
A+B \cos \left(2\left(\phi-\phi_{0}\right)\right)
$$

where $\phi$ is the angle between the spin moment in the $y z$ plane and the (111) normal. It is positive (negative) for a magnetic moment tilted above the upper (lower) terrace. The coefficients $A, B$ and $\phi_{0}$ are found by the usual Fourier-series technique.

The common property of all studied cases is that the energetically preferred easy axis of the magnetization is found to be parallel to the $x$-axis of the coordinate frame drawn in figure 2, similar to the ab initio results of Hong and $\mathrm{Wu}[8,9]$. To illustrate the physics behind this, let us consider an infinite free-standing Co chain oriented along the $z$-axis. The minorityspin electrons form a wide $\sigma$-band $\left(3 z^{2}-r^{2}\right.$ orbitals), doubly degenerate $\pi$-band $(x z, y z)$ and a narrow doubly degenerate $\delta$-band $\left(x y, x^{2}-y^{2}\right)$ [51-53]. Analogous features in the local density of states at the third $\mathrm{Co}$ atom in the $\mathrm{Co}_{7}$ chain are drawn in figure 4(a). $E_{\mathrm{F}}$ falls well inside a high-density peak in LDOS stemming from the $\delta$-orbitals with the magnetic orbital numbers $m= \pm 2$ and therefore an effective spin-orbit coupling is possible. LDOSs calculated with spin-orbit coupling are drawn in figure 4(b). The effect of spin-orbit coupling on the $\delta$-band is obvious.

Before proceeding to the discussion on the hard axis orientation, let us clarify the following point. The hard axis lying in the $x y$ plane and providing most of the energy for the whole system is determined by the Fourier fit of the MAE of the whole system. The angles found by an analogous fit of local MAE contributions from particular atoms need not coincide with the system hard axis direction. It is also true for extrema of angular moments on particular atoms [50]. This misorientation varies in a range from negligibly small values up to several degrees. Larger differences are possible due to numerical errors in fitting if the $B$ parameter in equation (1) is very small. The orientations of the hard axis in the $y z$ plane both for ideal and relaxed $\mathrm{Co}_{2}-\mathrm{Co}_{7}$ chains are demonstrated in figure 5. Relaxation has no significant effect on $\mathrm{Co}_{2}$ dimer. In both cases the angle between the hard axis and (111) normal is approximately $40^{\circ}$. Calculations performed for $\mathrm{Co}$ dimer on $\mathrm{Cu}(111)$ revealed an in-plane hard axis. A similar result was obtained by Lazarovits et al [54] for $\mathrm{Co}$ on $\mathrm{Ag}(001)$. The difference between the orientations of the hard axis of the Co dimer adsorbed at the step and on the flat surface is definitely the effect of the step $\mathrm{Cu}$ atoms. The effect of relaxation becomes very significant for the Co trimer. The hard axis orientation of the unrelaxed trimer is almost in-plane, but strong relaxation disturbs linear shape of the trimer (see figure 1) and makes hard axis to be almost 


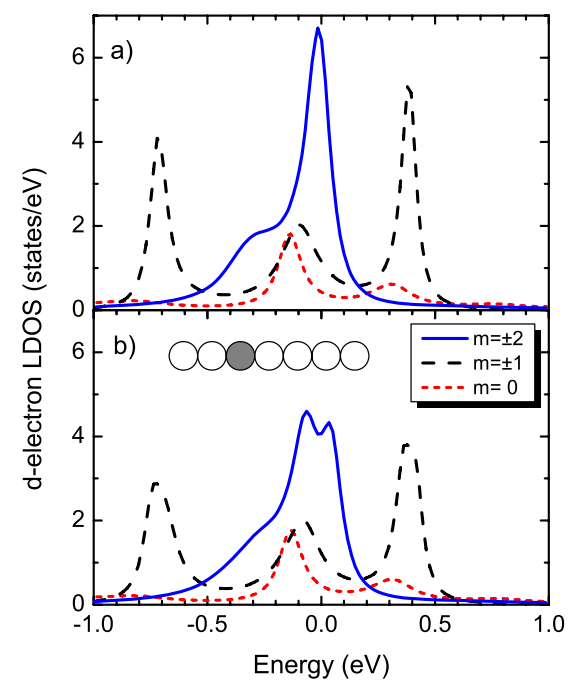

Figure 4. The effect of spin-orbit coupling on the d-electron LDOS at the third Co atom in the $\mathrm{Co}_{7}$ chain adsorbed at the step. (a) Spin-orbit coupling is neglected, (b) it is taken into account. The Fermi level coincides with the energy zero. Solid, long-dashed and short-dashed) curves correspond to the orbital moment eigenvalues $m= \pm 2, \pm 1,0$ with the quantization axis parallel to the $x$-axis of the coordinate frame drawn in figure 2.

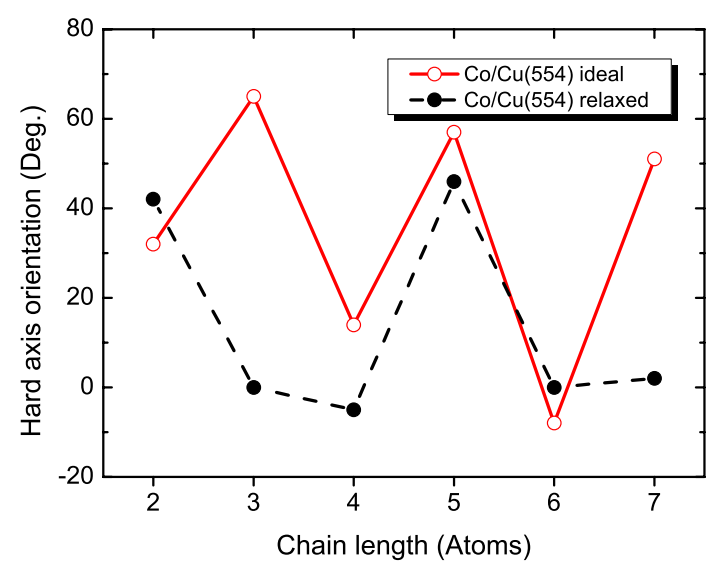

Figure 5. Orientation of the hard axis in the $y z$ plane for $\mathrm{Co}_{2}-\mathrm{Co}_{7}$ chains. Empty (full) circles correspond to ideal (relaxed) chains at the step. See the text for details.

parallel to the (111) normal. We check the direction of the hard axis of the Co trimer on the flat $\mathrm{Cu}(111)$ surface and found that it also points out-of-plane. The same result was obtained for Co trimer on $\mathrm{Ag}(001)$ [54]. The hard axis orientation of the $\mathrm{Co}_{4}, \mathrm{Co}_{5}, \mathrm{Co}_{6}$ are very similar for relaxed and ideal atomic positions but for the $\mathrm{Co}_{7}$ chain its orientation changes. Such a large difference between the relaxed and ideal $\mathrm{Co}_{7}$ can be explained by the very low anisotropy in the $y z$ plane for the relaxed chain.

Now we focus on the distribution of the magnetic properties along the chain and their averages. The total spin magnetic moments in the $\mathrm{Co}_{7}$ chain calculated by means of KKR Green's function can be found in table 1. The exhaustive information on MAE and orbital 
Table 2. MAE and magnetic orbital moments $L^{m}$ calculated for the ideal $\mathrm{Co}_{7}$ chain for the magnetization orientations along the easy, hard, and intermediate axis, respectively. Zero energy is chosen for magnetization orientation parallel to the surface (111) normal. Negative energy means that corresponding magnetization orientation is more energetically preferable. Easy magnetization axis (corresponding to the ground state) is oriented along the chain. Order of the Co atoms is illustrated in figure 3 . Average values $\langle\cdot\rangle$ per atom in the chain are also shown.

\begin{tabular}{llrrrrr}
\hline & & \multicolumn{5}{c}{ Co atom } \\
\cline { 3 - 6 } & Magn. orient. & \multicolumn{1}{c}{1} & \multicolumn{1}{c}{2} & \multicolumn{1}{c}{3} & \multicolumn{1}{c}{4} & $\langle\cdot\rangle$ \\
\hline MAE & Along the chain & -3.59 & -3.72 & -4.88 & -2.89 & -3.90 \\
$(\mathrm{meV})$ & $\phi=51^{\circ}$ & 0.14 & 0.11 & 0.31 & 0.06 & 0.17 \\
& $\phi=-39^{\circ}$ & -0.11 & -0.10 & -0.13 & -0.07 & -0.11 \\
\hline$L^{m}$ & Along the chain & 0.55 & 0.47 & 0.63 & 0.42 & 0.53 \\
$\left(\mu_{\mathrm{B}}\right)$ & $\phi=51^{\circ}$ & 0.28 & 0.22 & 0.19 & 0.27 & 0.23 \\
& $\phi=-39^{\circ}$ & 0.29 & 0.24 & 0.22 & 0.27 & 0.25 \\
\hline
\end{tabular}

Table 3. MAE and magnetic orbital moments $L^{m}$ calculated for the relaxed $\mathrm{Co}_{7}$ chain. For details see description of table 2 .

\begin{tabular}{|c|c|c|c|c|c|c|}
\hline & \multirow[b]{2}{*}{ Magn. orient. } & \multicolumn{4}{|c|}{ Co atom } & \multirow[b]{2}{*}{$\langle\cdot\rangle$} \\
\hline & & 1 & 2 & 3 & 4 & \\
\hline $\begin{array}{l}\text { MAE } \\
(\mathrm{meV})\end{array}$ & $\begin{array}{l}\text { Along the chain } \\
\phi=2^{\circ} \\
\phi=-88^{\circ}\end{array}$ & $\begin{array}{r}-3.23 \\
0.00 \\
0.05\end{array}$ & $\begin{array}{r}-3.80 \\
0.00 \\
0.02\end{array}$ & $\begin{array}{r}-5.05 \\
0.00 \\
-0.17\end{array}$ & $\begin{array}{r}-2.54 \\
0.00 \\
-0.41 \\
\end{array}$ & $\begin{array}{r}-3.81 \\
0.00 \\
-0.09 \\
\end{array}$ \\
\hline $\begin{array}{l}L^{m} \\
\left(\mu_{\mathrm{B}}\right)\end{array}$ & $\begin{array}{l}\text { Along the chain } \\
\phi=2^{\circ} \\
\phi=-88^{\circ}\end{array}$ & $\begin{array}{l}0.48 \\
0.25 \\
0.25\end{array}$ & $\begin{array}{l}0.40 \\
0.18 \\
0.18\end{array}$ & $\begin{array}{l}0.59 \\
0.24 \\
0.25\end{array}$ & $\begin{array}{l}0.39 \\
0.19 \\
0.24\end{array}$ & $\begin{array}{l}0.47 \\
0.22 \\
0.23\end{array}$ \\
\hline
\end{tabular}

moments for the same chain in ideal and relaxed configurations is listed in tables 2 and 3 respectively. The MAE and the orbital moments of the relaxed $\mathrm{Co}_{7}$ chain drop in comparison to the ideal system. We expect that the properties of chains will converge with damped oscillations as the chain length increases. Actually, we did some tests that allow us to compare unrelaxed infinite and $\mathrm{Co}_{7}$ chains on the ideal or stepped $\mathrm{Cu}(111)$ surface, respectively. The corresponding differences of the MAE, orbital moment or the hard axis orientation do not exceed several per cents. Orientation of hard axes is shown in figure 5. Stabilizations of the intermediate magnetization axis and easy axis with respect to the hard axis are demonstrated in figure 6 . For ideal chains the tendency towards stabilization is visible starting from $\mathrm{Co}_{4}$. The same trend is obtained for magnetic orbital moments (see figure 7). For relaxed chains a similar comparison is problematic since there is no effective way of $\mathrm{Co}-\mathrm{Co}$ distance relaxation for very long but finite chains. For example, for the $\mathrm{Co}_{7}$ chain, the $\mathrm{Co}-\mathrm{Co}$ bond length at the central part of the chain is relaxed only by $2 \%$. Naturally, broken-symmetry geometries should be checked for very long chains [51].

For short chains, there are marked oscillations of chain properties in dependence of their length. Such an oscillatory behavior, which has pronouncedly odd-even atomic length character (see figures 5-7), can be understood on intuitive grounds. Let us consider transformation of eigenstate orbital part (i.e. transformation of the spinor is not taken into account) with respect to the symmetry-plane reflection. For antisymmetric states and chains with odd number of atoms, the amplitude of the above two important $\delta$-orbitals $\left(x y, x^{2}-y^{2}\right)$ is zero on the central atom which has no analogy for even-atom chains. 


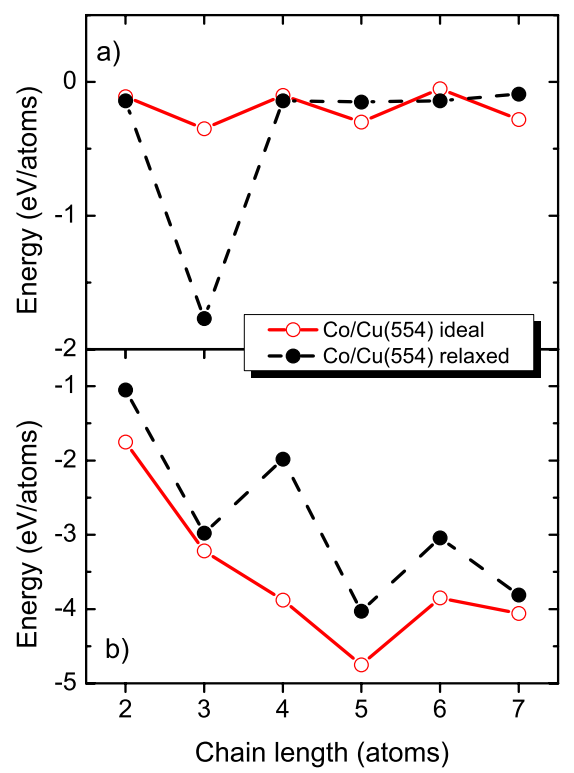

Figure 6. Stabilization of the intermediate magnetization axis (a) and easy axis (b) with respect to the hard axis (energy zero) demonstrated by the electronic part of the average magnetic anisotropy energy in the chain. Both in (a) and (b) panels empty (full) circles correspond to ideal (relaxed) chains at the step.

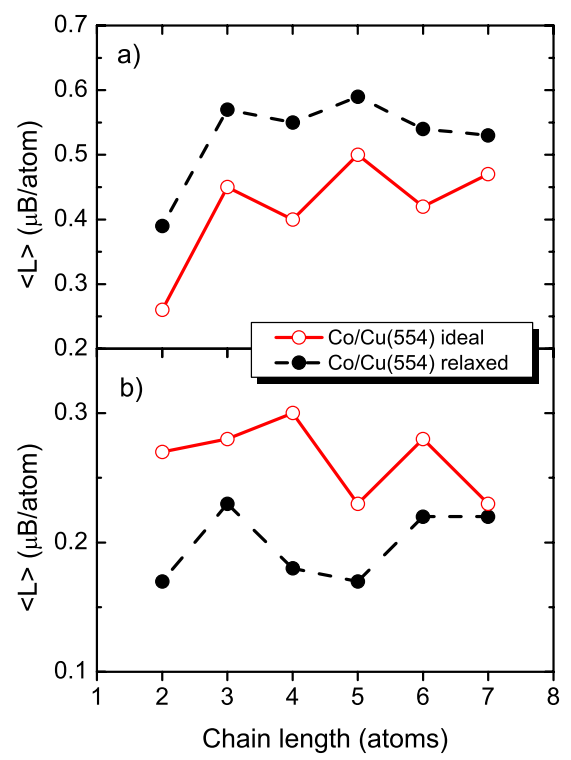

Figure 7. Projections of the magnetic orbital moment $L^{m}$ on the easy axis (a) and on the hard axis (b) determined by the Fourier fit of the whole chain according equation (1). Both in (a) and (b) panels empty (full) circles correspond to ideal (relaxed) chains at the step.

Finally, the changes of MAE and $L^{m}$ caused by a switch of magnetization direction can be connected by the approximate relation [55] involving the intra-atomic spin-orbit coupling 
parameter $\xi$ :

$$
\Delta E \approx-0.25 \xi \Delta L^{m}
$$

We use $\xi=0.06 \mathrm{eV}$ as in our previous studies [44]. As a rule, no serious deviations from equation (2) have been found. Equation (2) permits to qualitatively estimate the changes of the MAE.

\section{Conclusions}

We have demonstrated that the strain relief in the Co chains at a stepped $\mathrm{Cu}$ surface significantly affects the atomic structure of the chains and the morphology of the substrate. We have performed extensive calculations of the MAE for ideal and relaxed short Co chains. The easy axis is found to be aligned along the chains and the MAE is determined to be several meV per atom. The magnetic properties of chains exhibit oscillations with increasing chain length. Atomic relaxations are found to affect significantly the direction of the easy axis. We demonstrate also that relaxations reduce the MAE and magnetic moments of Co chains.

\section{Acknowledgments}

ŠP thanks the Max-Planck-Institut für Mikrostrukturphysik, Halle and the Martin-LutherUniversität, Halle-Wittenberg, for their kind hospitality. This work was supported by Deutsche Forschungsgemeinschaft DFG SPP 1165.

\section{References}

[1] Elmers H J, Hauschild J, Höche H, Gradmann U, Bethge H, Heuer D and Köhler U 1994 Phys. Rev. Lett. 73898

[2] Himpsel F J, Ortega J E, Mankey G J and Wills R E 1998 Adv. Phys. 47511

[3] Shen J, Skomski R, Klaua M, Jenniches H, Manoharan S S and Kirschner J 1997 Phys. Rev. B 562340

[4] Gambardella P, Dallmeyer A, Malti K, Malagoli M C, Eberhardt W, Kern K and Carbone C 2002 Nature 416301

[5] Dorantes-Dávila J and Pastor G M 1998 Phys. Rev. Lett. 81208

[6] Gambardella P, Dallmeyer A, Malti K, Malagoli M C, Rusponi S, Ohresser P, Eberhardt W, Carbone C and Kern K 2004 Phys. Rev. Lett. 93077203

[7] Vindigni A, Rettori A, Pini M C, Carbone C and Gambardella P 2006 Appl. Phys. A 82385

[8] Hong J and Wu R Q 2003 Phys. Rev. B 67020406

[9] Hong J and Wu R Q 2004 Phys. Rev. B 70060406

[10] Spišák D and Hafner J 2002 Phys. Rev. B 65235405

[11] Spišák D and Hafner J 2003 Phys. Rev. B 67214416

[12] Lazarovits B, Szunyogh L, Weinberger P and Újfalussy B 2003 Phys. Rev. B 68024433

[13] Lazarovits B, Szunyogh L and Weinberger P 2003 Phys. Rev. B 67024415

[14] Tischer M, Hjortstam O, Arvanitis D, Hunter Dunn J, May F, Baberschke K, Trygg J, Wills J M, Johansson B and Eriksson O 1995 Phys. Rev. Lett. 751602

[15] Felix-Medina R, Dorantes-Dávila J and Pastor G M 2002 New J. Phys. 4100.1

[16] Wierzbovska M, Delin A and Tosatti E 2005 Phys. Rev. B 72035439

[17] Pentcheva R and Scheffler M 2000 Phys. Rev. B 612211

[18] Dürr H A, van der Laan G, Vogel J, Panaccione G, Brookes N B, Dudzik E and McGrath R 1998 Phys. Rev. B 58 R11853

[19] Gambardella P, Rusponi S, Kern K, Brune H, Dhesi S S, Cabria I, Zeller R, Dederichs P, Dallmeyer A and Carbone C 2003 Science $\mathbf{3 0 0} 1130$

[20] Rusponi S, Cren T, Weiss N, Epple M, Buluschek P, Claude L and Brune H 2003 Nat. Mater. 2546

[21] Klautau A B and Frota-Pessǒa S 2004 Phys. Rev. B 70193407

[22] Lazarovits B, Újfalussy B, Szunyogh L, Stocks G M and Weinberger P 2004 J. Phys.: Condens. Matter 165833

[23] Nakagawa T, Watanabe H and Yokoyma T 2006 Phys. Rev. B 74134422

[24] Dhesi S S, van der Laan G, Dudzik E and Shick A B 2001 Phys. Rev. Lett. 87067201 
[25] Weber W, Back C H, Bischof A, Pescia D and Allenspach R 1995 Nature 374788

[26] Hahlin A, Karis O, Hunter Dunn J and Arvanitis D 2002 J. Appl. Phys. 916881

[27] Shick A B, Máca F and Oppeneer P M 2004 Phys. Rev. B 69212410

[28] Máca F, Shick A B, Redinger J and Oppeneer P M 2006 Czech. J. Phys. 5651

[29] Wildberger K, Stepanyuk V S, Lang P, Zeller R and Dederichs P H 1995 Phys. Rev. Lett. 75509

[30] Wildberger K, Zeller R and Dederichs P H 1997 Phys. Rev. B 5510074

[31] Bellini V, Papanikolaou N, Zeller R and Dederichs P H 2001 Phys. Rev. B 64094403

[32] Ignatiev P A, Stepanyuk V S, Klavsyuk A L, Hergert W and Bruno P 2007 Phys. Rev. B 75155428

[33] Cleri F and Rosato V 1993 Phys. Rev. B 4822

[34] Rosato V, Guillope B and Legrand B 1989 Phil. Mag. A 59321

[35] Levanov N A, Stepanyuk V S, Hergert W, Bazhanov D I, Dederichs P H, Katsnelson A A and Massobrio C 2000 Phys. Rev. B 612230

[36] Stepanyuk V S, Bazhanov D I, Baranov A N, Hergert W, Dederichs P H and Kirschner J 2000 Phys. Rev. B 6215398

[37] Stepanyuk V S, Klavsyuk A L, Hergert W, Saletsky A M, Bruno P and Mertig I 2004 Phys. Rev. B 70195420

[38] Pick Ś, Stepanyuk V S, Klavsyuk A L, Niebergall L, Hergert W, Kirschner J and Bruno P 2004 Phys. Rev. B 70224419

[39] Miron R A and Fichthorn K A 2004 Phys. Rev. Lett. 93128301

[40] Sastry K, Johnson D D, Goldberg D E and Bellon P 2005 Phys. Rev. B 72085438

[41] Lysenko O V, Stepanyuk V S, Hergert W and Kirschner J 2002 Phys. Rev. Lett. 89126102

[42] Miron R A and Fichthorn K A 2005 Phys. Rev. B 72035415

[43] Stepanyuk V S, Klavsyuk A L, Niebergall L, Saletsky A M, Hergert W and Bruno P 2005 Phase Transit. 7861

[44] Pick Š, Stepanyuk V S, Baranov A N, Hergert W and Bruno P 2003 Phys. Rev. B 68104410

[45] Pick Š 1998 Phys. Rev. B 571942

[46] Haydock R 1980 Solid State Phys. 35215

[47] Pick Š, Dorantes-Dávila J, Pastor G M and Dreyssé H 1993 Phys. Rev. B 50993

[48] Papaconstantopoulos D A 1986 Handbook of the Band Structure of Elemental Solids (New York: Plenum)

[49] Mackintosh A R and Andersen O K 1980 Electrons at the Fermi Surface ed M Springford (Cambridge: Cambridge University Press) p 149

[50] Shick A B, Máca F and Openeer P M 2004 Phys. Rev. B 69212410

[51] Spišák D and Hafner J 2002 Phys. Rev. B 65235405

[52] Kishi T, Kasai H, Nakanishi H, Diño W A and Komori F 2004 Surf. Sci. 566-588 1052

[53] Jin Y J, Kim I G and Lee J I 2004 Phys. Status Solidi b 2411431

[54] Lazarovits B, Szunyogh L and Weinberger P 2002 Phys. Rev. B 65104441

[55] Bruno P 1989 Phys. Rev. B 39865 